\title{
Composición química, contenido mineral y digestibilidad in vitro de raigrás (Lolium perenne) según intervalo de corte y época de crecimiento
}

\section{Chemical composition, mineral content and in vitro digestibility from ryegrass (Lolium perenne) in relation to the cutting interval and growing season}

\author{
Horacio Castro-Hernándeza , I gnacio Arturo Domínguez-Varaa* , Ernesto Morales-Almaráza , \\ Maximino Huerta-Bravob
}

\begin{abstract}
RESUMEN
El objetivo fue evaluar la calidad nutritiva del forraje raigrás (Lolium perenne) a diferente madurez (0, 7, 14 y 28 días) en tres épocas del año, otoño (O), invierno (I) y primavera-verano (PV), en un diseño experimental completamente al azar con arreglo factorial 4x3. La madurez del forraje aumentó de forma lineal y cuadrática el contenido de materia seca, fibra detergente ácido y lignina ácido detergente, y de forma cuadrática la fibra detergente neutro; la materia orgánica (MO) bajó de forma lineal y cuadrática, y la proteína cruda (PC) disminuyó linealmente al madurar el forraje. La MO y PC fueron mayores en PV ( $P<0.01)$. La producción total de gas, degradabilidad y producción de gas relativa, bajaron de forma lineal y cuadrática $(P<0.05)$ al madurar la planta; la degradabilidad y producción de gas relativa fueron mayores al día cero y en la época PV; el volumen de gas por efecto del intervalo de corte, en las tres épocas, disminuyó con la edad del forraje. El tiempo de retardo de incubación fue mayor $(P<0.01)$ en PV. El contenido mineral del forraje se afectó $(P<0.05)$ por el intervalo de corte y época del año; el mayor aporte fue al día cero y en PV. Los niveles de $\mathrm{P}, \mathrm{Ca}, \mathrm{K}, \mathrm{Mg}$ y Zn del forraje no cubren los requerimientos de vacas en producción en las distintas épocas y edades de corte evaluadas. El forraje tuvo mayor calidad en la época PV, antes de $\mathbf{2 8}$ días de edad.
\end{abstract}

PALABRAS CLAVE: Composición química, Época, Minerales, Digestibilidad, Madurez, Raigrás.

\begin{abstract}
The objective was to evaluate the nutritive quality of forage ryegrass (Lolium perenne) at different maturity $(0,7,14$ and 28 d) in three seasons of the year, autumn (A), winter (W) and spring-summer (SS) in an experimental design randomized with factorial treatments 4x3 arrangement. The maturity of the plant increased in linear and quadratic form the content of dry matter, acid detergent fiber and acid detergent lignin, and quadratic form of neutral detergent fiber; organic matter (OM) content fell in linear and quadratic form and the crude protein (CP) is reduced linearly by the maturity of the forage. The biggest contribution of OM and CP was in SS $(P<0.01)$. Total gas production, degradability and relative gas production, fell in linear and quadratic form $(P<0.05)$ with the maturity of the plant. Degradability and relative gas production were greater in the zero-day and at the season SS; the volume of gas produced by cutting interval effect, in three times, decreased with the age of the forage. The delay time of incubation was greatest $(P<0.01)$ in SS. The mineral concentration in the forage was affected $(P<0.05)$ by the range of cutting and the season of year; higher mineral content was in the zero-day cut and in SS. Concentrations of $\mathrm{P}, \mathrm{Ca}, \mathrm{K}, \mathrm{Mg}$, and $\mathrm{Zn}$ do not meet requirements for lactating cows at any seasons by maturity stage combination. The best forage quality was obtained in SS before $28 \mathrm{~d}$ of regrowth age.
\end{abstract}

KEY WORDS: Chemical composition, Minerals, Digestibility, Age, Season, Ryegrass.

Recibido el 19 de febrero de 2015. Aceptado el 24 de agosto de 2015.

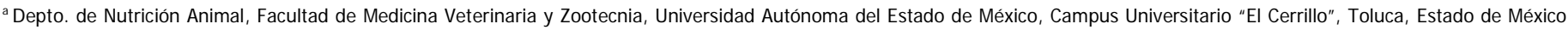
50090, tel. 722 2965542. México.

${ }^{b}$ Depto. de Zootecnia, Universidad Autónoma Chapingo, México.

*Autor de correspondencia: igy92@hotmail.com. 
La mayor limitante de la producción de rumiantes basada en forrajes es la fluctuación en su producción y calidad; esto determina en gran medida su respuesta para producción de leche y crecimiento, reflejándose en su consumo y digestibilidad; asimismo, el rendimiento, la composición botánica y calidad del forraje, son afectados por el manejo de la pradera, como la intensidad, la frecuencia y oportunidad de uso $^{(1)}$. El efecto del corte en el forraje depende de la cantidad y tipo de tejido removido, de su estado fenológico y condiciones meteorológicas al momento del corte; además, el efecto de la intensidad y frecuencia de corte en la tasa de crecimiento del forraje, y su acumulación, dependen de la duración del rebrote ${ }^{(2)}$.

El estudio de la frecuencia de corte, a 2, 4 y 6 semanas, en ballico perenne, indicó que el mayor rendimiento de materia orgánica (MO) digestible y proteína cruda (PC) se obtuvo al cortar el forraje cada cuatro semanas $^{(3)}$. El clima es determinante del crecimiento y rendimiento de las plantas; en invierno, con baja temperatura y menor radiación solar, el rendimiento del ballico perenne (cultivar) CV Barlatra disminuye notablemente, mientras que en primavera y verano tiene su máxima producción ${ }^{(3)}$. Por lo tanto, conocer en cada estación, la calidad del forraje, ante distintos intervalos de corte, en términos de su composición química, aporte mineral y de cinética digestiva, es la base para su uso eficiente $^{(4)}$ y representa una estrategia en el manejo óptimo del corte y en la planificación alimenticia complementaria para cubrir las necesidades nutricionales del rumiante durante el año.

La nutrición de rumiantes requiere de estudios más precisos sobre el valor nutricional del forraje, la sincronización entre la energía y el nitrógeno suministrados al rumen es una aproximación para mejorar la eficiencia de la fermentación; sin embargo, la aplicación de este concepto en el balanceo de dietas, según los nuevos programas de evaluación ${ }^{(5)}$, requiere de información cuantitativa sobre cinética digestiva para una estimación precisa de la cantidad de los nutrientes digeridos y de las propiedades intrínsecas del alimento que pueden limitar su disponibilidad $^{(6)}$. Las praderas constituyen la fuente más económica para la alimentación, por lo tanto, es importante conocer su calidad para aprovechar mejor el potencial productivo del animal. En el centro de México, en los sistemas de producción de leche, una opción para reducir costos es el uso de praderas de raigrás (Lolium perenne), entre otras. Su rendimiento y calidad están influenciados por el efecto estacional y la edad de la planta(3,7).

La calidad de las praderas cultivadas es uno de los factores determinantes de los sistemas de producción de leche en pequeña escala en el altiplano central de México(8); en estos sistemas, el rango de producción es de 15 a $19 \mathrm{~kg}$ de leche por vaca por día(9); en este contexto, la ingestión de proteína, grasa, fibra soluble, etc., que aportan energía, así como los minerales, influirán considerablemente sobre el rendimiento animal; varios minerales esenciales ( $\mathrm{Ca}, \mathrm{P}$, $\mathrm{K}, \mathrm{Na}, \mathrm{Mg}, \mathrm{Se}, \mathrm{Zn}, \mathrm{I}, \mathrm{Cu}, \mathrm{Mo}, \mathrm{Co}, \mathrm{Mn}$ ) afectan directa o indirectamente el desempeño productivo animal ${ }^{(10)}$. Por ello, es necesario mantener la calidad del forraje que cubra los requerimientos del rumiante ${ }^{(11)}$. Mantener una pradera tierna, con menos desarrollo estructural y más contenido celular digestible ${ }^{(11)}$ es dave para optimizar su valor nutricional ${ }^{(12)}$. Otros autores consignan que bajos valores de energía metabolizable (EM), PC y digestibilidad de la materia orgánica (DMO) ocurren en una edad avanzada de la planta(13).

El objetivo de la investigación fue evaluar el efecto de cuatro frecuencias de corte, en tres épocas del año, sobre la calidad nutritiva del forraje raigrás en función del contenido de materia seca (MS), MO, PC, fibra detergente neutro (FDN), fibra detergente ácido (FDA), lignina ácido detergente (LAD), digestibilidad in vitro por producción de gas y contenido mineral, en el Valle de Toluca, Estado de México.

El trabajo se llevó a cabo en las praderas del módulo de bovinos productores de leche, y en los laboratorios de Bromatología y metabolismo del Departamento de Nutrición Animal de la Facultad de Medicina Veterinaria y Zootecnia, de la Universidad Autónoma del Estado de México, localizada en el centro oeste del Estado, a 19o 42' 16" N y 99 o 39'38" O a 2,638 msnm. Clima templado frío con lluvias en primavera y verano, con precipitación pluvial anual de 800 a $1,000 \mathrm{~mm}^{(14)}$.

Se obtuvieron muestras de una pradera de raigrás, con una superficie aproximada de 2 ha. Se usó la misma pradera para las tres épocas experimentales y las cuatro edades de corte; en los periodos de descanso, la pradera se pastoreó por 
bovinos lecheros en producción. Se aplicó riego rodado cada 15 días con agua de un pozo profundo, excepto durante julio a septiembre por la precipitación pluvial. La pradera no se fertilizó 30 días previos al primer corte, ni en los demás muestreos en las tres épocas evaluadas. En los periodos que no se muestreó, se aplicaron $100 \mathrm{~kg} / \mathrm{ha}$ de urea ( $\mathrm{N}, 46 \%)$ cada 28 días.

La pradera se dividió en cinco sub parcelas (lotes rectangulares), identificadas con tiras de nylon y estacas de madera, cuyas medidas fueron $3.5 \mathrm{~m}$ de largo y $1.5 \mathrm{~m}$ de ancho. En cada lote se hicieron cuatro cortes de forraje al azar, el primero al día 0 , para conocer el estado nutrimental inicial de la pradera, el resto a los 7, 14 y 28 días, bajo el método de muestreo destructivo( ${ }^{(15)}$, en tres épocas del año, otoño (octubre), con temperatura media de $12.6^{\circ} \mathrm{C}$ y precipitación media de $54 \mathrm{~mm}$; invierno (diciembre) con temperatura media de $10.1^{\circ} \mathrm{C}$ y precipitación media de $8.3 \mathrm{~mm}$; y al final de la primavera y principio de verano (junio-julio) con temperatura media de $13.8^{\circ} \mathrm{C}$ y precipitación media de $148 \mathrm{~mm}^{(16)}$. El total de muestras de forraje por época fue de 20, y de 60 para todo el experimento.

Las muestras de forraje se secaron a $60{ }^{\circ} \mathrm{C}$, durante $24 \mathrm{~h}$, después se molieron en molino de malla de $1 \mathrm{~mm}$ y se almacenaron en frascos hasta su análisis en el laboratorio. Se determinó MS, MO, $P C^{(17)}, F D N$, FDA y $L A D^{(18)}$.

La fermentación de la MS se realizó mediante la técnica de producción de gas con incubación de frascos de vidrio ${ }^{(19)}$. El líquido ruminal se obtuvo de dos bovinos fistulados en rumen (peso vivo $450 \pm$ $15 \mathrm{~kg}$ ), alimentados dos veces por día (0800 y 1600 h) con $1.5 \mathrm{~kg}$ de heno de alfalfa, $2.5 \mathrm{~kg}$ de rastrojo de maíz y $1.25 \mathrm{~kg}$ de concentrado [12\% PC, 11.5 mega jouls (MJ) de EM]. El líquido ruminal se obtuvo por la mañana, con los animales en ayuno (16 h) antes de suministrar el alimento, a través de la fístula. El líquido de cada animal se colocó en dos termos y se llevó de inmediato al laboratorio, donde se filtró a través de dos capas de gasa y finalmente fue mezclado. Aproximadamente $800 \mathrm{mg}$ de MS de muestra se incubaron por duplicado con $90 \mathrm{ml}$ de solución buffer y $10 \mathrm{ml}$ de líquido ruminal(19). Para el estudio de las diferencias entre épocas de muestreo, se realizaron tres series por inoculación, utilizando la media de los dos frascos de cada muestra en cada incubación. En cada serie de incubación, dos frascos adicionales sin sustrato y otros dos con forraje de digestibilidad conocida (estándar) se utilizaron para conocer la producción de gas y se incluyeron como blanco y estándar, respectivamente, para las correspondientes correcciones. El volumen de gas producido se registró a las 3, 6, 9, 12, 24, 36, 48, 60,72 y 96 h de incubación. Después del período de incubación, el residuo de fermentación de cada frasco se recuperó y secó a $60^{\circ} \mathrm{C}$ durante $48 \mathrm{~h}$ para estimar la proporción de MS desaparecida (MSd). La producción de gas relativa (PGR; $\mathrm{ml}$ gas/g MS desaparecida a las 96 h) se calculó de los volúmenes de gas registrados ${ }^{(20)}$. Para estimar la evolución de la fermentación microbiana, la acumulación de producción de gas se calculó de acuerdo al siguiente modelo(21):

$y=A\left\{1-\exp \left[-b^{(t-T)}-c^{(v t-v T)}\right]\right\}$, donde $y=$ producción de gas acumulada $(\mathrm{ml}), \mathrm{t}=$ tiempo de incubación ( $h$ ), $A=$ asíntota de la curva (producción de gas total, $\mathrm{ml}), \mathrm{b}\left(\mathrm{h}^{-1}\right)$ y $\mathrm{c}\left(\mathrm{h}^{1 / 2}\right)=$ constantes de producción de gas, $\mathrm{T}=$ "lag time" $(\mathrm{h})$, tiempo para iniciar la degradación del alimento por los microorganismos del rumen.

Para medir el contenido mineral en el forraje se incineró $1 \mathrm{~g}$ de cada muestra, y posteriormente se sometió a digestión con ácido clorhídrico para su posterior análisis ${ }^{(12)}$. El $P$ se determinó por colorimetría ${ }^{(22)}$ y el resto $(\mathrm{Ca}, \mathrm{Na}, \mathrm{K}, \mathrm{Mg}, \mathrm{Cu}, \mathrm{Fe}, \mathrm{Zn})$ por espectrofotometría de absorción atómica con flama ${ }^{(23)}$.

Las variables dependientes de composición química, digestibilidad in vitro y concentración mineral del forraje se procesaron mediante análisis de varianza, para un diseño experimental completamente al azar con arreglo factorial $4 \times 3$ de tratamientos (edades $x$ épocas); se usó un modelo mixto que consideró la edad de corte (efecto fijo), la parcela o unidad experimental (efecto aleatorio) y la época del año como mediciones repetidas a través del tiempo en cada parcela o unidad experimental (UE). Para evaluar los efectos lineal, cuadrático y cúbico de la edad de corte del forraje en las variables dependientes se realizó un análisis de polinomios ${ }^{(24)}$. La comparación de medias se hizo por la prueba de Tukey ${ }^{(25)}$. 
La calidad nutrimental del forraje raigrás fue afectada $(P<0.01)$ por la edad de corte, la época del año y la interacción edad de corte con época; la madurez de la planta aumentó de forma lineal y cuadrática el contenido de MS, FDA y LAD, y de forma cuadrática el de FDN; mientras que el contenido de MO bajó de forma lineal y cuadrática y el de PC se redujo de forma lineal por la madurez del forraje. Lo anterior sugiere que con intensidades de corte de 7 y 14 días (una a dos semanas), la defoliación es muy intensa y el tiempo muy corto, por lo tanto no permite recuperar las reservas de azúcares en raíces y tallos, en consecuencia, hay menor rebrote por la escasa área foliar y menor fotosíntesis ${ }^{(26)}$. El mayor aporte de MO y PC del forraje $(P<0.01)$ fue en la época de primaveraverano (Cuadro 1 ), lo cual es importante, ya que en esta época es cuando se produce de 65 a $70 \%$ del total anual (8-13 t MS/ha), y en otoño e invierno de 30 a $35 \%$ restante ${ }^{(3)}$. Lo anterior se atribuye a que en las épocas de primavera y verano se presentan las mayores precipitaciones, temperaturas y radiaciones solares, mientras que en otoño e invierno baja mucho la cantidad de lluvia y las temperaturas son menores con presentación de heladas ${ }^{(27)}$.

El promedio de PC para las tres épocas fue mayor a $140.0 \mathrm{~g} / \mathrm{kg} \mathrm{MS}$, y cubre el requerimiento de vacas lecheras con producción de $13 \mathrm{~kg}$ de leche por día(28). Los resultados coinciden con los de otros estudios en forrajes similares, en los que se evaluó el estado de madurez ${ }^{(3,29)}$ y la época del año ${ }^{(3,30)}$. La disminución de la calidad nutritiva del forraje evaluado podría afectar el consumo de MS para cubrir los requerimientos nutricionales de vacas lecheras con mayor producción (16,31); lo anterior debido al aumento de los carbohidratos estructurales de la planta con reducción del contenido de proteína y energía en la misma.

La fermentación in vitro del forraje se afectó $(P<0.05)$ por la edad de corte, época del año e interacción en sus distintos parámetros (Cuadro 2). La producción total de gas (parámetro A), la

Cuadro 1. Efecto de la edad de corte y época del año en la composición química ( $\mathrm{g} / \mathrm{kg} \mathrm{MS}$ ) del forraje raigrás (Lolium perenne) cultivado en el valle de Toluca, México

\begin{tabular}{|c|c|c|c|c|c|c|c|c|c|c|c|c|c|}
\hline & \multicolumn{4}{|c|}{ Edad de corte (días) } & \multirow[b]{2}{*}{ EEM } & \multicolumn{3}{|c|}{ Época del año } & \multirow[b]{2}{*}{ EEM } & \multicolumn{3}{|c|}{ Efectos de: } & \multirow[b]{2}{*}{ Polinomio } \\
\hline & 0 & 7 & 14 & 28 & & Otoño & Invierno & Pri-Ver & & Edad & Época & EdadxÉpoca & \\
\hline MS & $890.8^{c}$ & $901.6^{b}$ & $909.1^{a}$ & $907.1^{\mathrm{ab}}$ & 2.852 & $901.4^{b}$ & $910.3^{a}$ & $895.6^{b}$ & 4.804 & $\star *$ & ** & ** & L, C \\
\hline MO & $862.7^{a}$ & $806.5^{b}$ & $720.0 c$ & $782.1^{b}$ & 17.878 & $785.4^{\mathrm{ab}}$ & $776.2^{b}$ & $816.9^{a}$ & 28.75 & ** & * & ** & L, C \\
\hline FDN & $484.1^{b}$ & $498.1^{\mathrm{ab}}$ & $528.9^{a}$ & $489.2^{b}$ & 18.018 & $473.4^{b}$ & $497.2^{b}$ & $529.5^{a}$ & 21.03 & ** & ** & * & $C$ \\
\hline FDA & $279.4^{c}$ & $306.4^{b}$ & $343.7^{a}$ & $304.1^{b}$ & 11.492 & $294.9 b$ & $309.1^{\mathrm{ab}}$ & $321.3^{a}$ & 14.23 & ** & ** & ** & $L, C$ \\
\hline
\end{tabular}

EEM= error estándar de la media; $\mathrm{MS}=$ materia seca; $\mathrm{MO}=$ materia orgánica; $\mathrm{PC}=$ proteína cruda; $\mathrm{FDN}=$ fibra detergente neutra; $\mathrm{FDA}=$ fibra detergente ácida; $\mathrm{LAD}=$ lignina ácido detergente. ${ }^{1}$ Polinomio para edad de corte: $\mathrm{L}=$ lineal, $\mathrm{C}=$ cuadrático.

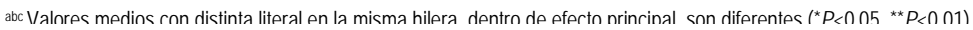

Cuadro 2. Efecto de la edad de corte y época del año en los parámetros de fermentación in vitro (producción de gas) del forraje raigrás (Lolium perenne) cultivado en el valle de Toluca, México

\begin{tabular}{|c|c|c|c|c|c|c|c|c|c|c|c|c|c|}
\hline & \multicolumn{4}{|c|}{ Edad de corte (días) } & \multirow[b]{2}{*}{ EEM } & \multicolumn{3}{|c|}{ Época del año } & \multirow[b]{2}{*}{ EEM } & \multicolumn{3}{|c|}{ Efectos de: } & \multirow[b]{2}{*}{ Polinomio $^{1}$} \\
\hline & 0 & 7 & 14 & 28 & & Otoño & Invierno & Pri-Ver & & Edad & Época & EdadxÉpoca & \\
\hline$A$ & $230.0^{a}$ & $189.9^{b}$ & $168.7^{b}$ & $189.0^{\mathrm{b}}$ & 10.800 & $186.6^{b}$ & $179.8^{b}$ & $212.8^{a}$ & 10.820 & ** & $\star \star$ & ** & L, C \\
\hline B & $0.065^{a}$ & $0.067^{a}$ & $0.069^{a}$ & $0.073^{a}$ & 0.0005 & $0.072^{\mathrm{a}}$ & $0.067^{a b}$ & $0.066^{b}$ & 0.0005 & ns & * & ns & ns \\
\hline$c$ & $0.065^{a}$ & $-0.063^{a}$ & $-0.068^{a}$ & $0.062^{\mathrm{a}}$ & 0.0181 & $-0.066^{a b}$ & $-0.065^{a}$ & $-0.073^{b}$ & 0.0114 & ns & ** & * & ns \\
\hline Lag time & $1.372^{\mathrm{a}}$ & $1.214^{\mathrm{a}}$ & $1.265^{a}$ & $1.073^{a}$ & 0.4035 & $1.150^{\mathrm{b}}$ & $1.050^{\mathrm{b}}$ & $1.490^{\mathrm{a}}$ & 0.1731 & * & ** & * & ns \\
\hline Deg.(g/100gMS) & $77.54^{\mathrm{a}}$ & $70.41^{b}$ & $64.34^{c}$ & $71.03^{b}$ & 2.3889 & $71.14^{\mathrm{a}}$ & $67.47^{b}$ & $73.90^{\mathrm{a}}$ & 2.863 & ** & ** & ** & L, C \\
\hline
\end{tabular}

$\mathrm{EEM}=$ error estándar de la media.

${ }^{1}$ Polinomio para edad de corte: $L=$ =lineal, $C=$ cuadrático.

$A=$ producción total de gas ( $\mathrm{ml}$ gas/g MS inicial); $\mathrm{B}=$ tasa de fermentación $\left(\mathrm{h}^{-1}\right) ; \mathrm{c}=$ tasa de fermentación $\left(\mathrm{h}^{-12}\right)$; lag time, tiempo de retardo de incubación $\left(\mathrm{h}^{-1}\right)$; Deg= Degradabilidad $(\mathrm{g} / 100 \mathrm{~g}$ MS); PGR, producción de gas relativa ( $\mathrm{mL}$ gas/g MS desaparecida a $96 \mathrm{~h}$ ); ns= no significativa.

abc Valnrec medinc s.nn dictinta literal en la misma hilera dentrn de efectn nrincinal snn diferentec $(* P<\cap \cap 5 * * P<\cap \cap 1)$ 
degradabilidad y la producción de gas relativa, se redujeron de forma lineal y cuadrática $(P<0.05)$ con la madurez de la planta; la degradabilidad y la producción de gas relativa fueron mayores en el día cero y en la época primavera-verano; el volumen de gas producido por la fermentación del forraje por efecto del intervalo de corte, en las tres épocas, disminuyó con la edad del mismo (Figura 1). Una disminución en la producción de gas (A) con el incremento de la madurez de la planta es esperada debido a una reducción en la degradabilidad de la pared celular(32), por lo tanto, si el volumen de gas liberado como producto de la fermentación microbiana es menor, en consecuencia hay menor producción molar de los metabolitos acetato y butirato(19); debido a que la fermentación del sustrato hasta propionato produce gas sólo desde la neutralización del ácido, por consiguiente, una menor producción de gas es asociada con la fermentación propiónica(33).

El análisis de polinomios mostró que las tasas de fermentación B ( $\mathrm{h}-1)$ y c ( $\mathrm{h}-1 / 2)$, y el tiempo de retardo de incubación (lag time) no se afectaron ( $P>0.05$ ) por la edad de la planta. El valor de tiempo de retardo de incubación fue mayor $(P<0.01)$ en la época primavera-verano (1.490 h); esto puede estar asociado con el mayor contenido de FDN y FDA observado en la misma época, causando un retraso en el comienzo de la degradación del forraje por parte de los microorganismos del rumen. La información generada sobre la fase temprana de fermentación es importante para identificar los pequeños cambios que ocurren en el peso del sustrato durante las primeras horas de incubación ${ }^{(34)}$; la energía para el crecimiento de los microorganismos del rumen deriva de la fermentación de los carbohidratos, principalmente almidón y celulosa, cuya digestión anaerobia produce ácidos grasos volátiles (AGV) acético, propiónico y butírico, succinato, formato, lactato, etanol, $\mathrm{CO}_{2}$, metano y cantidades trazas de $\mathrm{H}_{2}$; también aportan esqueletos carbonados esenciales para la síntesis de biomasa microbiana ${ }^{(35)}$. La producción de gas derivada de la fermentación de proteínas es relativamente pequeña, y la derivada de la grasa es insignificante ${ }^{(36)}$. Las condiciones anaeróbicas $\left(\mathrm{CO}_{2}\right)$ y el ambiente más reducido promueve un menor tiempo de colonización y una tasa de digestión de la FDN más rápida( ${ }^{(37)}$.

La digestibilidad in vitro de la MS (DIVMS) en las variedades barlatra, cropper y talbot de forraje Lolium perenne cultivado de mayo a agosto fue de 711,744 y $766 \mathrm{~g} / \mathrm{kg}^{(38)}$. En un estudio realizado bajo condiciones climáticas similares a las del presente trabajo, en donde se evaluó la digestibilidad in vitro de una pradera mixta de tres gramíneas (Lolium perenne, Festuca arundinacea y Dactylis glomerata) y dos leguminosas (Medicago sativa y Trifolium repens), en diferentes épocas del año, reportaron valores $(\mathrm{g} / \mathrm{kg}$ de MS) para la época de primavera y verano de 660, valores inferiores a los reportados en esta investigación para la época primavera-verano (729); para la época de otoño reportaron valores de degradabilidad de 630 y para invierno de 660; estos valores son inferiores a los reportados en esta investigación ( 711 para otoño y $684 \mathrm{~g} / \mathrm{kg}$ de MS para invierno $)^{(7)}$. Otros autores ${ }^{(8)}$ reportan que la digestibilidad in vitro ( $\mathrm{g} / \mathrm{kg}$ de MS) del pasto Lolium perenne es mayor $(P<0.05)$ en invierno $(690)$ que en verano (640) u otoño y primavera (628), con una disminución $(P<0.05)$ lineal conforme avanza la madurez de la planta $(2,4$ y 6 semanas de edad). Estos resultados difieren de los del presente trabajo en cuanto a la época del año, pero con una semejanza por el efecto de la edad de corte.

Figura 1. Dinámica del volumen de gas producido por la fermentación del forraje raigrás ( $\mathrm{ml}$ de gas/g MS inicial) según el intervalo de corte (día cero o, día $7 \mathbf{\square}$, día $14 \Delta$ y día $28 \square$ ) en tres épocas del año

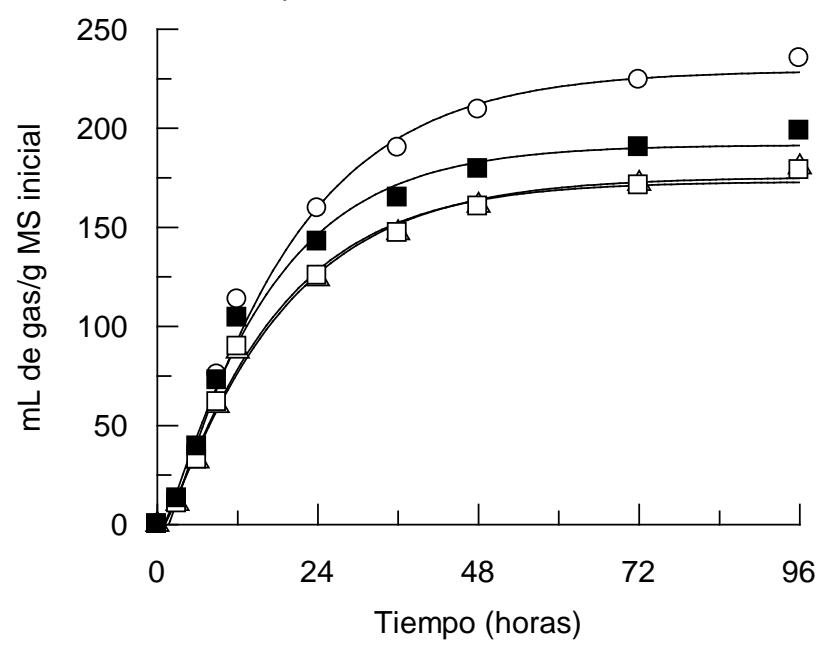


El contenido de FDN y FDA determinan el contenido de celulosa y hemicelulosa de la pared celular vegetal, junto con la lignina, por lo tanto, la colonización por los microrganismos del rumen, de las estructuras vegetales y su posterior degradación, está íntimamente relacionada con la composición y estructura de la pared celular ${ }^{(39)}$. La lignina frecuentemente es mencionada como una limitante de la digestión de la fibra; sin embargo, algunas investigaciones sugieren que el contenido de lignina no sería responsable de la disminución de la digestión de la fibra, sino que la acción de la lignina consiste en reducir el acceso de las enzimas hidrolíticas a la fibra digestible. Otros estudios mencionan efectos tóxicos de la lignina sobre los microorganismos del rumen, lo que inhibe su adhesión a las partículas vegetales y su actividad fermentativa ${ }^{(31)}$. Los componentes solubles son rápidamente fermentados poco después de iniciar la incubación, subsecuentemente, ocurre un cambio hacia la fermentación de las fracciones insolubles, las cuales requieren ser hidratadas y colonizadas por los microorganismos ruminales antes de ser fermentados, y por último, el gas se produce por el reciclaje de la población microbiana, mas no por la fermentación del alimento ${ }^{(40)}$. Los componentes solubles en detergentes neutros (SDN) comprenden azúcares simples, y sus polímeros de cadena corta, sustancias pécticas y alimidones, una fracción no carbohidratada, que incluye proteínas, polifenoles solubles, cenizas solubles, ácidos orgánicos y lípidos. Los SDN son importantes en la etapa temprana de la fermentación del sustrato (forraje), pero menos significativos en etapas posteriores ${ }^{(41)}$.

Los sustratos con alta degradabilidad verdadera pero, proporcionalmente a la cantidad de sustrato, con baja producción de gas, presentan mayor consumo de MS, mayor producción de masa microbiana, mayor eficiencia en la síntesis de proteína microbiana, menor producción de metano y menor producción de $\mathrm{AGV}^{(36,42)}$. Lo anterior es importante en la predicción del consumo voluntario (CV) de los alimentos, ya que el CV de los forrajes está mejor correlacionado con sus características de degradabilidad ruminal, que con la digestibilidad en el tubo digestivo total; y la producción de gas a partir de la FDN tiene mejor correlación con el CV que los valores de incubación del forraje entero(43), debido a que el consumo de pared celular es el responsable de la distensión de la pared ruminal ${ }^{(34)}$. Por lo tanto, en rumiantes, la tasa de digestión fraccional se ha usado para predecir el CV de forrajes ${ }^{(6)}$, pero para mejorar su precisión se requiere incorporar información relacionada con el ambiente, la especie, el estado nutricional y nivel de producción del animal( $^{(44)}$.

La concentración de minerales en el forraje raigrás cultivado en el valle de Toluca se afectó $(P<0.05)$ por el intervalo de corte, la época del año y su interacción. La mayor concentración de todos los minerales fue en la época de primavera-verano, y para todos lo macro minerales y el Cu fue en el día cero de corte (Cuadro 3).

Cuadro 3. Efecto de la edad de corte y época del año en el contenido mineral (\% y ppm BS) del forraje raigrás (Lolium perenne) cultivado en el valle de Toluca, México

\begin{tabular}{|c|c|c|c|c|c|c|c|c|c|c|c|c|c|c|c|}
\hline & \multicolumn{4}{|c|}{ Edad de corte (días) } & \multirow[b]{2}{*}{ EEM } & \multicolumn{3}{|c|}{ Época del año } & \multirow[b]{2}{*}{ EEM } & \multicolumn{3}{|c|}{ Efectos de: } & \multirow[b]{2}{*}{ Polinomio ${ }^{1}$} & \multirow[b]{2}{*}{$\mathrm{Nc}^{2}$} & \multirow[b]{2}{*}{$\operatorname{Req}^{3}$} \\
\hline & 0 & 7 & 14 & 28 & & Otoño & Invierno & Pri-Ver & & Edad & Época & EdxÉp & & & \\
\hline$P$ & $0.31^{a}$ & $0.21^{b}$ & $0.15^{b}$ & $0.17^{b}$ & 0.0349 & $0.24^{a}$ & $0.10^{\mathrm{b}}$ & $0.30^{\mathrm{a}}$ & 0.0665 & ** & 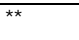 & ** & $\mathrm{L}$ & 0.25 & 0.33 \\
\hline $\mathrm{Ca}$ & $0.14^{a}$ & $0.11^{\mathrm{ab}}$ & $0.09^{b}$ & $0.11^{\mathrm{ab}}$ & 0.0217 & $0.11^{\mathrm{ab}}$ & $0.09 \mathrm{~b}$ & $0.14^{a}$ & 0.0329 & ns & * & ns & ns & 0.30 & 0.51 \\
\hline $\mathrm{Na}$ & $0.21^{a}$ & $0.15^{b}$ & $0.11^{c}$ & $0.13^{\mathrm{bc}}$ & 0.3742 & $0.15^{\mathrm{a}}$ & $0.13^{a}$ & $0.17^{a}$ & 0.0377 & * & ns & * & $\mathrm{L}, \mathrm{C}$ & 0.06 & 0.18 \\
\hline $\mathrm{Mg}$ & $0.15^{\mathrm{a}}$ & $0.12^{\mathrm{ab}}$ & $0.10^{\mathrm{b}}$ & $0.12^{\mathrm{ab}}$ & 0.0184 & $0.13^{a}$ & $0.11^{\mathrm{a}}$ & $0.13^{a}$ & 0.0214 & * & ns & ns & $L, C$ & 0.20 & 0.20 \\
\hline Ca:Py & $0.54^{b}$ & $0.71^{\mathrm{ab}}$ & $0.87^{a}$ & $0.86^{\mathrm{a}}$ & 0.1331 & $0.70^{\mathrm{b}}$ & $1.02^{\mathrm{a}}$ & $0.52^{b}$ & 0.1839 & * & ** & ns & L & 1:1 & $2: 1$ \\
\hline $\mathrm{Fe}$ & $122.1^{b}$ & $232.5^{\mathrm{a}}$ & $322.1^{\mathrm{a}}$ & $243.0^{a}$ & 51.059 & $209.7^{a}$ & $254.4^{\mathrm{a}}$ & $25.7^{\mathrm{a}}$ & 58.852 & ** & ns & ** & $L, C$ & 30.0 & 50.0 \\
\hline $\mathrm{Zn}$ & $41.5^{c}$ & $47.8^{a}$ & $51.8^{a}$ & $62.9^{a}$ & 11.261 & $17.6^{c}$ & $51.9^{b}$ & $83.4^{a}$ & 10.574 & * & ** & ** & ns & 30.0 & 40.0 \\
\hline
\end{tabular}

EEM= error estándar de la media.

1 Polinomio para edad de corte: $L=$ =lineal, $C=$ cuadrático

2 Nivel crítico $(14,19,31,55)$.

${ }^{3}$ Requerimiento para una vaca Holstein de $400 \mathrm{~kg}$ de PV con producción de $13 \mathrm{~kg}$ leche/día(31).

abc Valores medins r.nn distinta literal en la misma hilera dentro de efec.tn nrinc.inal son diferentes $\left({ }^{*} P<0 \cap .5{ }^{*} P<0 \cap 01\right)$ 
Fósforo $(\mathrm{P})$. El contenido de $\mathrm{P}$ bajó de forma lineal $(P<0.05)$ con la madurez de la planta; en invierno bajó drásticamente su concentración, lo que coincide con el descenso de la temperatura ambiental y menor presencia de lluvias ${ }^{(15)}$; además de la reducción natural del $\mathrm{P}$ en la planta por mayor madurez ${ }^{(45)}$. En esta investigación, el contenido de $P$ del forraje, en su mayoría, no cubre el requerimiento de una vaca lechera en producción $(0.33 \%)^{(28)}$. La concentración de $\mathrm{P}$ encontrada es inferior a lo informado por otros autores, quienes evaluaron el efecto de la época del año( ${ }^{(30)}$ o la edad de corte ${ }^{(29)}$. En cuanto a la época del año, investigaciones anteriores en la misma región con forraje raigrás ${ }^{(46)}$ reportan mayor contenido de $\mathrm{P}$ al encontrado en el presente estudio $(0.44 \%$ en época seca y $0.36 \%$ durante las lluvias). Estudios realizados en gramíneas de zonas cálidas informaron concentraciones de 0.06 y $0.07 \%$ para las época seca y lluviosa, respectivamente ${ }^{(47)}$.

Calcio (Ca). El Ca fue afectado $(P<0.05)$ por la edad de la planta y la época del año, pero no por la interacción o análisis polinomial ( $P>0.05)$. La mayor concentración de Ca $(P<0.05)$ se observó en el día cero y en la época primavera-verano y el menor contenido en invierno. Contrario a lo observado en este estudio, el $\mathrm{Ca}$ suele aumentar cuando la temperatura es baja(11). Los valores de Ca en el forraje, en las tres épocas y cuatro intervalos de corte, fueron deficientes $(<0.11 \%)$ y no cubren lo que requiere una vaca en producción $(0.51 \%$ Ca) ${ }^{(28)}$; asimismo, son menores a lo informado por otros autores en forraje raigrás de la misma región ${ }^{(45)}$. En México el Ca está considerado deficiente en los forrajes a nivel nacional ${ }^{(47)}$.

Relación Ca:P. La relación fue afectada $(P<0.05)$ por los efectos principales y aunque aumentó de forma lineal con la madurez del forraje, no logró una relación 2:1, considerada adecuada para el ganado(28). En vacas lecheras la nutrición adecuada de $C a, P$ y vitamina $D$, es esencial para evitar problemas de hipocalcemia.

Potasio (K). El contenido de $\mathrm{K}$ se redujo de forma lineal y cuadrática por efecto de la edad del forraje. El descenso en la temperatura ambiental y la menor precipitación pluvial, que ocurren normalmente en otoño e invierno, pueden limitar el crecimiento de la planta y su eficiencia para absorber los nutrientes del suelo ${ }^{(17,48)}$. Se observaron deficiencias de $K$ en el forraje en la época de invierno y en los días 7 y 14 de edad, por lo que no se cubrieron los requerimientos de una vaca en producción ${ }^{(28)}$. La mayoría de los forrajes son adecuados en $\mathrm{K}$, pero bajo ciertas condiciones, como la aplicación de fuentes de NNP y el exceso de Mg, pueden reducir su contenido a través del año.

Sodio (Na). La concentración de Na disminuyó $(P<0.05)$ de forma lineal y cuadrática con la madurez de la planta. En forrajes templados el aumento del $\mathrm{K}$ tiende a disminuir el contenido de $\mathrm{Na}$, aunque no se observaron deficiencias de $\mathrm{Na}$ en este estudio, pese a las altas concentraciones de $\mathrm{K}$. El contenido de $\mathrm{Na}$ del forraje sólo cubrió los requerimientos de vacas en producción (13 kg leche/día) en el día cero de edad y en la época primavera-verano ${ }^{(28)}$. Otros autores reportan concentraciones de $\mathrm{Na}$ de $0.11 \%$ para la misma región $^{(46)}$. En el ganado lechero es baja la incidencia de carencia de $\mathrm{Na}^{(28)}$.

Magnesio (Mg). El contenido de Mg disminuyó $(P<0.05)$ de forma lineal y cuadrática con la madurez del forraje, con deficiencias en las tres épocas y cuatro edades de corte $(<0.2 \%)$; esto pudo deberse al alto nivel de $K$, ya que reduce la absorción de $\mathrm{Mg}$ en la planta(49).

Cobre (Cu). El contenido de Cu en el forraje sólo se afectó $(P<0.01)$ por la interacción edad de corte con época, pero no hubo deficiencias en ningún intervalo de corte y época del año, por lo que sí se cubrió el requerimiento de vacas lecheras con producción ${ }^{(28)}$. En la zona se han detectado deficiencias de $\mathrm{Cu}$ en el suelo, planta y ovinos en pastoreo, asociadas a un exceso natural de $\mathrm{Fe}$ debido al origen del material parental volcánico en los suelos del valle de Toluca, así como por el pH ácido del suelo(50-52); sin embargo, en el presente trabajo el forraje raigrás no tuvo deficiencia de $\mathrm{Cu}$, pero sí exceso de Fe. El contenido medio de $\mathrm{Cu}$ en el forraje fue alto (22.2 vs 13.5 ppm) y mayor al reportado por otros autores ${ }^{(46)}$.

Hierro (Fe). El contenido de Fe se afectó $(P<0.01)$ por la edad de corte e interacción edad de corte con época; aumentó de forma lineal y cuadrática con la madurez del forraje; su 
concentración indicó exceso de Fe (> 120 ppm). Este exceso de Fe en el forraje puede deberse a la acidez del suelo, favoreciendo la disponibilidad y absorción del mismo mineral por la planta(51); además, los suelos de esta zona son ricos en Fe ( $>$ de $255 \mathrm{ppm})^{(52)}$. Las concentraciones de Fe cubren los requerimientos de vacas con producción de leche de $13 \mathrm{~kg}$ leche/día ${ }^{(28)}$. A pesar de las altas concentraciones de Fe reportadas, no se observaron deficiencias de $\mathrm{Cu}$, probablemente porque las deficiencias de $\mathrm{Cu}$ en el ganado se han reportado con valores de 250 a 500 ppm de Fe, los cuales son superiores a los reportados en esta investigación ${ }^{(52)}$. Los contenidos de Fe encontrados en este trabajo son inferiores a los observados en otros forrajes de clima templado(52) con valores medios de 550 ppm. El contenido medio de Fe observado en el forraje de este estudio es inferior al reportado en otros trabajos (230 vs 550 ppm) ${ }^{(52)}$ y superior al reportado en climas cálidos (230 vs 131 ppm).

Zinc ( $Z n)$. El contenido se afectó $(P<0.05)$ por la edad de corte, la época del año y su interacción, pero el análisis polinomial del intervalo de corte no tuvo efecto alguno ( $P>0.05)$. Su contenido en el forraje sólo en otoño estuvo por debajo del nivel crítico ${ }^{(28)}$, por lo que no cubre el requerimiento de una vaca en producción ${ }^{(28)}$; en contraste, los valores de las otras épocas fueron superiores a los indicados por otros autores ${ }^{(46,52)}$ en forrajes templados. Se ha informado que un nivel alto de Ca y Fe en el forraje puede afectar la absorción de Zn, aunque esto no se presentó en invierno y primavera-verano(28).

Se concluye que la calidad nutrimental del forraje raigrás cultivado en el valle de Toluca, en términos de su composición química, digestibilidad y aporte de minerales, disminuyó con la madurez de la planta. El intervalo de corte aumentó el contenido de MS, FDN, FDA y LAD, y disminuyó la concentración de MO y PC. En la época de primavera y verano el forraje fue más digestible y tuvo mayor aporte de MO, PC y minerales. La madurez de la planta redujo la producción total de gas, la degradabilidad y la producción de gas relativa; el volumen de gas producido por la fermentación del forraje por efecto del intervalo de corte, en las tres épocas, disminuyó con la edad del mismo. La concentración de minerales en el forraje disminuyó con la madurez de la planta; el mayor contenido para todos los minerales fue en primavera y verano, y para todos los macro minerales y el $\mathrm{Cu}$ fue en el corte inicial. Los niveles de P, Ca, K, Mg y Zn en el forraje no cubren los requerimientos de una vaca en producción láctea moderada en las distintas épocas y edades de corte evaluadas.

\section{AGRADECIMIENTOS}

A la Universidad Autónoma del Estado de México, UAEM por el financiamiento para realizar esta investigación a través del proyecto con clave $1747 / 2003$.

\section{LITERATURA CITADA}

1. Hernández-Garay A, Matthew C, Hodgson J . Effect of spring grazing management of perennial ryegrass and ryegrass-white clover pastures. 1. Tissue turnover and herbage accumulation. N ZJ Agr Res 1997; 40:25-35.

2. Richards $\mathrm{JH}$. Physiology of plants recovering from defoliation. Proc XVII Int Grassland Cong. N Z and Australia 1993;85-94.

3. Velasco-Zebadúa ME, Hernández GA, González HV. Rendimiento y valor nutritivo del Ballico perenne (Lolium perenne) en respuesta a la frecuencia de corte. Téc Pecu Méx 2005;43(1):247-258.

4. Posada SL, Noguera RR. Técnica in vitro de producción de gases: una herramienta para la evaluación de alimentos para rumiantes. Liv Res Rural Develop 2005;17(4):450-465.

5. Russel JB, O`Connor JD, Fox DG, Van Soest PJ, Sniffen CJ. A net carbohydrate and protein system for evaluating cattle diets: I. Ruminant fermentation. J Anim Sci 1992; 70:3551-3561.

6. López S, Carro MD, González JS, Ovejero FJ. Comparison of different in vitro and in situ methods to estimate the extent and rate of degradation of hays in rumen. Anim Feed Sci Tech 1998; 73:99-113.

7. Lemus RV, Lugo LG, Valencia GE, Villagrán VB. Desempeño de una pradera irrigada en clima templado, establecida para el pastoreo con bovinos lecheros. Vet Méx 2002;33(1):11:26.

8. Arriaga JC, Espinoza OA, Albarrán PB, Castelán OO. Producción de leche en pastoreo de praderas cultivadas: Una alternativa para el altiplano central. Ciencia Ergo Sum 1999;6:290-300.

9. Álvarez A, Cervantes F, Espinoza A. Características del sistema lácteo y sus principales tendencias en México, en: Agroindustria rural y territorio. Nuevas tendencias en el análisis de la lechería. Álvarez A, et al, editores. UAEM, Toluca, México. 2007(2):15-52.

10. Minson DJ. Forages in ruminant nutrition. 1st ed. USA: Academic Press; 1990.

11. Hodgson J, Brookes IM. Nutrition of grazing animals. Chapter 9. In: White J, Hodgson J editors. N Z Pastures Crop Sci. Oxford Univ Press; 1999: 117-132. 
12. Hodgson J. Grazing management: science into practice. Longman handbooks in agriculture. New York, Longman Scientific and Technical and J ohn Wiley; 1990.

13. Machado CF, Morris ST, Hodgson J, Fathalla M. Seasonal changes of herbage quality within a New Zealand beef cattle finishing pasture. N ZJ Agr Res 2005;48:265-270.

14. INEGI. Instituto Nacional de Estadística, Geografía e Informática. Prontuario de información geográfica municipal de los Estados Unidos Mexicanos. México. 2009.

15. McDowell LR, Conrad JH. Trace mineral nutrition in Latin America. World Anim Rev 1977;6(24):24:33.

16. INEGI. Instituto Nacional de Estadística, Geografía e Informática. Anuario estadístico. México. 2004.

17. AOAC. Official methods of analysis. $15^{\text {th }}$ ed., Arlington, VA, USA: Association of Official Analytical Chemists. 1990.

18. Van Soest PJ, Robertson JB, Lewis BA. Methods for dietary fiber, neutral detergent fiber and nonstarch polysaccharides in relation to animal nutrition. J Dairy Sci 1991;74(10):3583-3597.

19. Theodorou KM, Williams AB, Dhanoa SM, McAllan BA, France J. A simple gas production method using a pressure transducer to determine the fermentation kinetics of ruminant feeds. Anim Feed Sci Technol 1994;48(3):185-197.

20. González-Ronquillo M, Fondevila M, Barrios-Urdaneta A, Newman Y. In vitro gas production from buffel grass (Cenchrus ciliaris $L$.) fermentation in relation to the cutting interval, the level of nitrogen fertilization and the season of growth. Anim Feed Sci Tech 1998; 72:19-32.

21. France J, Dhanoa MS, Theodorou MK, Lister SJ, Davies DR, I saac D. A model to interpret gas accumulation profiles associated with in vitro degradation of ruminant feeds. J Theor Biol 1993; 163(1): 99-111.

22. Harris WD, Popat P. Determination of the phosphorus content of lipids. J Am Oil Chem Soc 1954; 31(4):124-127.

23. Fick KR, McDowell LR, Wilkinson NS, Funk DJ, Conrad JH, Valdivia R. Métodos de análisis de minerales para tejidos de plantas y animales. Florida, USA: Departamento de Ciencia Animal, Universidad de Florida; 1979.

24. SAS. SAS/STAT User's Guide: Statistics (version 8). Cary NC. USA: SAS Inst. Inc. Statistical Analysis System Institute. 1999.

25. Steel RGD, Torrie JH. Principles and procedures of statistics: a biometrical approach. 2nd ed. New York, USA: Mcgraw-Hill Book Co; 1980 .

26. Matthew C, Hodgson J. Form and function of grass [CD ROM]. Grassview Inst Nat Resources. Massey University, New Zealand. 1997.

27. García E. Modificaciones al sistema de clasificación climática de Köppen. 4aํ ed. México: Universidad Nacional Autónoma de México; 1988.

28. NRC. National Research Council. Nutrient requirements of dairy cattle. 7th ed. Washington, DC, USA: National Academic Press; 2001.

29. Lozano Al, Rodríguez HSA, Díaz SH, Rodríguez FJM, Fernández BJ M, Narváez MJM. Producción de forraje y calidad nutritiva en mezclas de triticale ( $\mathrm{X}$ Triticosecale Wittmack) y ballico anual
(Lolium multiflorum L.) en Navidad, NL. Tec Pecu Méx 2002; 40(1):17-35

30. Mosquera-Lozada MR, González-Rodríguez A, Rigueiro-Rodríguez A. Sward quality affected by different grazing pressures on dairy systems. J Range Manage 2000;53(6):603-610.

31. Jung AG, Allen MS. Characteristics of plants cell walls affecting intake and digestibility of forages by ruminants. J Anim Sci 1995; 73:2774-2790.

32. Ribeiro J r GO, Teixeira AM, Velasco FO, Faria Júnior WG, Pereira LGR, Chaves AV, et al. Production, nutritional quality and in vitro methane production from Andropogon gayanus grass harvested at different maturities and preserved as hay or Silage. Asian Austral J Anim Sci 2014;27(3):330-341.

33. Getachew $\mathrm{G}$, Blummel $\mathrm{M}$, Makkar $\mathrm{H}$, Becker $\mathrm{K}$. In vitro measures techniques for assesment of nutritional quality of feeds: review. Anim Feed Sci Tech 1998; 72:261-281.

34. Rosero JR. Estudio químico "in situ", "in vitro" e microscópico da parede celular de cinco genotipos de sorgo colhidos em três epocas de corte [Ph. D. Thesis]. Bello Orizonte: Esola de Veterinária da Universidade Federal de Minas Gerais; 2002.

35. Schofield P, Pitt RE, Pell AN. Kinnetics and fiber digestion from in vitro gas production. J Anim Sci 1994; 72:2980-2991.

36. Makkar $\mathrm{H}$. Recent advances in in vitro gas method for evaluation of nutritional quality of feed resources. 2001. http://www.fao.org/ DOCREP/ARTICLE/AGRIPPA/570_EN_toc.htm. Consultado 8 Sep, 2015.

37. Grant RJ, Mertens DR. Impact of in vitro fermentation techniques upon kinetics of fiber digestion. J Dairy Sci 1992; 75:1263-1272.

38. Pérez PJ, González MSS, Abarca BJ A. Rendimiento y digestibilidad de la materia seca de variedades de Lolium perenne $L$. con tres presiones de pastoreo. Agrociencia 1997;31:37-43.

39. Valenciaga D, Chongo B. La pared celular. Influencia de su naturaleza en la degradación microbiana ruminal de forrajes. Rev Cub Cienc Agríc 2004;48(4):343-350.

40. Cone WJ, Van Gelder AH, Driehuis F. Description of gas production profiles with a three-fasic model. Anim Feed Sci Technol 1997;66:31-45

41. Stefanon B, Pell AN, Schofield P. Effect of maturity on digestion kinetics of water-soluble and water-insoluble fractions of alfalfa and brome hay. J Anim Sci 1996; 74:1104-1115.

42. Blümmel M, Mgomezulu R, Chen XB, Makkar HP, Becker K, Orskov ER. The modification of an in vitro gas production test to detect roughage related differences an in vivo microbial protein synthesis as estimated by the excretion of purine derivatives. J Agric Sci 1999; 133:335-340.

43. Blümmel N, Becker $K$. The degradability characteristics of fifty-four roughages of roughage neutral-detergent fibers as described by in vitro gas production and their relationship to voluntary feed intake. British J Nutr 1997; 77:757-768.

44. Pell AN, Doane PH, Schofield P. In vitro digestibility and gas production. Simposio sobre Tópicos Speciais em Zootecnia, Lavras MG, 1997:109-132.

45. Suttle NF. Mineral nutrition of livestock. 4th ed. UK: CABI ; 2010.

46. Morales AE, Domínguez-Vara I, González-Ronquillo M, J aramillo EG, Castelán OO, Pescador SN, Huerta BM. Diagnóstico mineral en 
forrajes y suero sanguíneo de bovinos lecheros en dos épocas en el Valle central de México. Téc Pecu Méx 2007;45(3):329-344.

47. Huerta BM. Nutrición mineral de rumiantes en pastoreo. Curso alternativas de manejo de bovinos carne en pastoreo. Universidad Autónoma Chapingo. Chapingo, México 1997:19-72.

48. McDowell LR, Valle G. Major minerals in forages. In: Givens DI, et al. editors. Forage evaluation in ruminant nutrition. 1st ed. London, UK: CABI; 2000:373-397.

49. Robinson D, Kappel LC, Boling JA. Management practices to overcome the incidence of grass tetany. J Anim Sci 1989;67:3470-3484.
50. Humphries WR, Bremner I, Phillippo $M$. The influence of dietary iron on copper metabolism in the calf. In: Mills CF, et al. editors. Trace elements in man and animals. Aberdeen, Scotland: Commonwealth Agric Bureaux; 1985:371-373.

51. McDowell LR, Houser RH, Fick KR. Iron, manganese and zinc in ruminant nutrition. Conrad J H, McDowell LR editors. Latin Am Symp Mineral Nutr Res Grazing Rum. Florida, USA: Universidad de Florida; 1978:108-116.

52. Domínguez-Vara I, Huerta BM. Concentración e interrelación mineral en suelo, forraje y suero de ovinos durante dos épocas en el valle de Toluca, México. Agrociencia 2008;42(2):173-183. 